\title{
SOME OBSERVATIONS ON THE SECOND NORMAL FORM
}

\author{
LUONG CAO SON, VU DUC THI
}

\begin{abstract}
For functional dependency, the second normal form (2NF) which was introduced by E. F. Codd has been widely investigated both theoretically and practically. In this paper, we give a new necessary and sufficient condition for an arbitrary relation scheme is in $2 \mathrm{NF}$ and its set of minimal keys is a given Sperner system.
\end{abstract}

\section{INTRODUCTION}

Now we start with some necessary definitions, and in the next section we formulate our results. Definition 1. Let $r=\left\{h_{1}, \ldots, h_{n}\right\}$ be a relation over $R$, and $A, B \subseteq R$. Then we say that $B$ functionally depends on $A$ in $r$ (denoted $A \underset{r}{\stackrel{f}{\longrightarrow}} B$ ) iff

$$
\left(\forall h_{i}, h_{j} \in r\right)(\forall a \in A)\left(h_{i}(a)=h_{j}(a)\right) \Rightarrow(\forall b \in B)\left(h_{i}(b)=h_{j}(b)\right) .
$$

Let $F_{r}=\{(A, B): A, B \subseteq R, A \underset{r}{\stackrel{f}{\longrightarrow}} B\} . F_{r}$ is called the full family of functional dependencies of $r$. Where we write $(A, B)$ or $A \rightarrow B$ for $A \stackrel{f}{\underset{r}{\longrightarrow}} B$ when $r, f$ are clear from the next context.

Definition 2. A functional dependency (FD) over $R$ is a statement of the form $A \rightarrow B$, where $A, B \subseteq R$. The FD $A \rightarrow B$ holds in a relation $r$ if $A \underset{r}{\stackrel{f}{\longrightarrow}} B$. We also say that $r$ satisfies the FD $A \rightarrow B$.

Definition 3. Let $R$ be a finite set, and denotes $P(R)$ its power set. Let $Y \subseteq P(R) \times P(R)$. We say that $Y$ is an $f$-family over $R$ iff for all $A, B, C, D \subseteq R$

(1) $(A, A) \in Y$,

(2) $(A, B) \in Y,(B, C) \in Y \Rightarrow(A, C) \in Y$,

(3) $(A, B) \in Y, A \subseteq C, D \subseteq B \Rightarrow(C, D) \in Y$,

(4) $(A, B) \in Y,(C, D) \in Y \Rightarrow(A \cup C, B \cup D) \in Y$.

Clearly, $F_{r}$ is an $f$-family over $R$. $F_{r}=\dot{Y}$

It is known [1] that if $Y$ is an arbitrary $f$-family, then there is a relation $r$ over $R$ such that

Definition 4. A relation scheme $s$ is a pair $\langle R, F\rangle$, where $R$ is a set of attributes, and $F$ is a set of FDs over $R$. Let $F^{+}$be a set of all FDs that can be derived from $F$ by the rules in Definition 3 .

Clearly, in [1] if $s=\langle R, F\rangle$ is a relation scheme, then there is a relation $r$ over $R$ such that $F_{r}=F^{+}$. Such a relation is called an Armstrong relation of $s$.

Definition 5. Let $r$ be a relation, $s=\langle R, F\rangle$ be a relation scheme, $Y$ be an $f$-family over $R$, and $A \subseteq R$. Then $A$ is a key of $r$ (a key of $s$, a key of $Y$ ) if $A \underset{r}{\stackrel{f}{\longrightarrow}} B\left(A \rightarrow R \in F^{+},(A, R) \in Y\right)$. $A$ is a minimal key of $r(s, Y)$ if $A$ is a key of $r(s, Y)$ and any proper subset of $A$ is not a key of $r(s, Y)$. Denote $K_{r},\left(K_{s}, K_{Y}\right)$ the set of all minimal keys of $r(s, Y)$.

Clearly, $K_{r}, K_{s}, K_{Y}$ are Sperner systems over $R$. 
Definition 6. Let $K$ be a Sperner system over $R$. We define the set of antikeys of $K$, denote by $K^{-1}$, as follows:

$$
K^{-1}=\{A \subset R:(B \in K) \Rightarrow(B \not \subset A) \text { and }(A \subset C) \Rightarrow(\exists B \in K)(B \subseteq C)\} .
$$

It is easy to see that $K^{-1}$ is also a Sperner system over $R$.

It is known [4] that if $K$ is an arbitrary Sperner system plays the role of the set of minimal keys (antikeys), then this Sperner system is not empty (does't contain $R$ ).

Definitions 7. Let $I \subseteq P(R), R \in I$, and $A, B \in I \Rightarrow A \cap B \in I$. Let $M \subseteq P(R)$. Denote $M^{+}=\left\{\cap M^{\prime}: M^{\prime} \subseteq M\right\}$. We say that $M$ is a generator of $I$ iff $M^{+}=I$. Note that $R \in M^{+}$but not in $M$, since it is the intersection of the empty collection of sets.

Denote $N=\left\{A \in I: A \neq \cap\left\{A^{\prime} \in I: A \subset A^{\prime}\right\}\right\}$.

In [6] it is proved that $N$ is the unique minimal generator of $I$. Thus, for any generator $N^{\prime}$ of $I$ we obtain $N \subseteq N^{\prime}$.

Definition 8. Let $r$ be a relation over $R$, and $E_{r}$ the equality set of $r$, i.e. $E_{r}=\left\{E_{i j}: 1 \leq i<j \leq\right.$ $|R|\}$, where $E_{i j}=\left\{a \in R: h_{i}(a)=h_{j}(a)\right\}$. Let $T_{R}=\left\{A \in P(R): \exists E_{i j}=A\right.$, no $\left.\exists E_{p q}: A \subset E_{p q}\right\}$. Then $T_{R}$ is called the maximal equality system of $r$.

Definition 9. Let $r$ be a relation, and $K$ a Sperner system over $R$. We say that $r$ represents ' $K$ if $K_{r}=K$.

The following theorem is known in [10]

Theorem 1. Let $K$ be a relation, and $K$ a Sperner system over $R$. $r$ presents $K$ iff $K^{-1}=T_{r}$, where $T_{r}$ is the maximal equality system of $r$.

From Theorem 1 we obtain the following corollary.

Corollary 1. Let $s=\langle R, F\rangle$ be a relation scheme and $r$ a relation over $R$. We say that $r$ represents $s$ if $K_{r}=K_{s}$. Then $r$ represents $s$ iff $K_{s}^{-1}=T_{r}$, where $T_{r}$ is the maximal equality system of $r$.

In [9] we proved the following theorem.

Theorem 2. Let $r=\left\{h_{1}, \ldots, h_{m}\right\}$ be a relation, and $F$ and $f$-family over $R$. Then $F_{r}=F$ iff for every $A \in P(R)$.

$$
H_{F}(A)= \begin{cases}\bigcap_{A \subseteq E_{g}} E_{g} & \text { if } \exists E_{g}: A \subseteq E_{g}, \\ R & \text { otherwise, }\end{cases}
$$

where $H_{F}(A)=\{A \in R:(A,\{a\}) \in F\}$ and $E_{r}$ is the equality set of $r$.

Definition 10. Let $s=\langle R, F\rangle$ be a relation scheme over $R$. We say that an attributet $a$ is prime if belong to a minimal key of $s$, and nonprime otherwise. Then $s=\langle R, F\rangle$ is in $2 \mathrm{NF}$ if $K^{\prime} \rightarrow\{a\} \notin F^{+}$ for each $K \in K_{s}, K^{\prime} \subset K$, and $a$ is nonprime.

If a relation scheme is changed to a relation we have the definition of $2 \mathrm{NF}$ for relation.

Definition 11. [5] Let $P$ be a set of all $f$-families over $R$. An ordering over $P$ is defined as follows: For $F, F^{\prime} \in P$ let $F \leq F^{\prime}$ iff for all $A \subseteq R, H_{F^{\prime}}(A) \subseteq H_{F}(A)$, where $H_{F}(A)=\{a \in R$ : $(A,\{a\}) \in F\}$.

Theorem 3. [9] Let $K$ be a Sperner system over $R$. Let:

$$
L(A)=\left\{\begin{array}{ll}
\bigcap_{A \subseteq B} B & \text { if } \exists B: A \subseteq B, \\
R & \text { otherwise, }
\end{array}\right. \text {. }
$$


and $F=\{(C, D): D \subseteq L(C)\}$.

Then $F$ is an $f$-family over $R, H_{F}=L$, and $K_{F}=K$. If $F^{\prime}$ is an arbitrary $f$-family over $R$ such $K_{F^{\prime}}=K$ then $F \leq F^{\prime}$ holds.

\section{RESULTS}

Now we present a new necessary and sufficient condition for an arbitrary relation scheme is in $2 \mathrm{NF}$ and its set of minimal keys is a given Sperner system.

Let $s=\langle R, F\rangle$ be a relation scheme over $R$. From $s$ we construct $Z(s)=\left\{X^{+}: X \subseteq R\right\}$, and compute the minimal generator $N_{s}$ of $Z(s)$. We put

$$
T_{s}=\left\{A \in N_{s}: \nexists B \in N_{s}, A \subset B\right\}
$$

It is know [1] that for a given relation scheme $s$ there is a relation $r$ such that $r$ is an Armstrong relation of $s$. On the other hand, by Corollary 1 and Theorem 2 the following proposition is clear.

Proposition 1. Let $s=\langle R, F\rangle$ be a relation scheme over $R$. Then:

$$
K_{s}^{-1}=T_{s} .
$$

Let $K$ be a Sperner system over $R$. Denote $T\left(K^{-1}\right)=\left\{A: \exists B \in K^{-1}, A \subseteq B\right\}$ and $K_{n}=\{a \in$ $R:$ no $\exists A \in K, a \in A\} . K_{n}$ is called the set of nonprime attributes of $K$.

Theorem 4. Let $s=\langle R, S\rangle$ be a relation scheme and $K$ a Sperner system over $R$. Denote $M(K)=$ $\{A-a: a \in A, A \in K\}$. Set $I^{*}=\left\{B: B=C^{+}, C \in M(K)\right\}$ and $I_{s}=\left\{B-a:, a \in K_{n}, B \in I^{*}\right\}$, where $K_{n}$ is the set of nonprime attributes of $K$.

Then $s$ is in $2 N F$ and $K_{s}=K$ if and only if

$$
\{R\} \cup K^{-1} \cup I_{s} \subseteq\{R\} \cup T\left(K^{-1}\right) .
$$

Proof. Assume that $s$ is in $2 \mathrm{NF}$ and $K=K_{s}$. From definitions of $Z(s), T\left(K^{-1}\right)$ and by Proposion 1 we obtain $Z(s) \subseteq\{R\} \cup T\left(K^{-1}\right)$. It is easy to see that if $K_{n}=\emptyset$ then $\{R\} \cup K^{-1} \cup I_{s} \subseteq Z(s)$. Assume that $K_{n} \neq \emptyset$. According to Proposition $1 ; K_{s}=K$ and by definition of $Z(s)$ we have $\{R\} \cup K^{-1} \subseteq Z(s)$. If there is a $B \in I^{*}$ and a $K_{n}: B-a \notin Z(s)$. Thus, $B-a \subset(B-a)^{+}$ holds. From definition of $I^{*}$ there is a $C \in M(K): C^{+}=B$. Clearly, $a \in(B-a)^{+}$holds. According to definition of closure we obtain $(B-a)^{+}=C^{+}=B$. Hence, $a \in C^{+}$holds. Thus, $C \rightarrow\{a\} \in F^{+}, a \notin C$ hold. This conflicts with the fact that $s$ is in 2 NF. Consequently, we obtain $\{R\} \cup K^{-1} \cup I_{s} \subseteq Z(s)$.

Now, assume that we have $(*)$. By definitions of $Z(s), K^{-1}, T\left(K^{-1}\right)$, and by Proposition 1 we obtain $K_{s}=K$. If $K_{n}=\emptyset$ then $s$ is in 2FN. Assume that $K_{n} \neq \emptyset$. Suppose that there is a $D \subset A$. $A \in K_{s}(1)$ and $a \in K_{n}, a \notin D$, but $D \rightarrow\{a\} F^{+}$. By (1) and according to constructions of $M(K)$ and $I^{*}$ there is a $C \in M(K): D \subset C$. $I_{s}$ is obvious that $a \notin C$.

Clearly, $D^{+} \subseteq C^{+}$and $a \subseteq C^{+}$. Set $B=C^{+}$. It can be seen that $C \subseteq B-a$. Consequently, $B-a \subset C^{+}=(B-a)^{1}$. This conflicts with the fact that $I_{s} \subseteq Z(s)$. Thus, $s$ is in 2NF. The proof is complete.

\section{REFERENCES}

[1] Armstrong W.W., Dependency Structures of Database Relationships, Information Processing 74, Holland Publ. Co., 1974, p. 580-583.

[2] Beeri C., Bernstein P. A., Computational problems related to the design of normal form relational schemes, ACM Trans on Database Syst. 4 (1) (1979) 30-59.

[3] Beeri C., Dowd M., Fagin R., Staman R., On the structure of Armstrong relations for functional dependencies, J. ACM 31 (1) (1984) 30-46. 
[4] Bernstein P.A., Synthesizing third normal form relations from functional dependencies, $A C M$ Trans. on Database Synt. 1 (1976) 277-298.

[5] Burosch G., Demetrovics J., Katona G. O., The poset of closures as a model of changing databases, Order 4 (1987) 127-142.

[6] Demetrovics J., On the equivalence of candidate keys with Sperner system, Acta Cybernetica 4 (1979) 247-252.

[7] Demetrovics J., Logical and structural investigation of relational datamodel, MTA - SZTAKI Tanulmanyok, Budapest, 114 (1980) 1-97.

[8] Demetrovics J., Hencsey G., Libkin L. O., Muchik I. B., Normal form relation scheme: a new characterization, Manuscript.

[9] Demetrovics J., Thi V.D., Some results about functional dependencies, Acta Cybernetica Hungary 8 (3) (1988) 273-278.

[10] Demetrovics J., Thi V. D., Relations and minimal keys, Acta Cybernetica Hungary 8 (3) (1998) 279-285.

[11] Demetrovics J., Thi V.D., On keys in the relation datamodel, Inform. Process Cybern. EIK 24 (10) (1988) 515-519.

[12] Demetrovics J., Thi V.D. Normal form and minimal keys in the relational datamodel, Acta Cybernetica $11(3)$ (1994) 205-215.

[13] Demetrovics J., Thi V.D., Some results about normal forms for functionla dependency in the ralational datamodel, Discrete Applied Mathematics 69 (1996) 61-74.

[14] Gottlob G., Libkin L., Investigations on Armstrong relations, dependency inference, and exclused functional dependencies, Acta Cybernical Hungary 7 (4) (1990) 385-402.

[15] Lucchesi C.L., Osbors S.L., Candidate keys for relations, J. Comput. Syst. Scien. $17(2)$ (1978) 270-279.

[16] Maier D., Minimum cover in the relational database model, JACM 27 (4) (1980) 664-674.

[17] Mannila H., Raika K. J., Design by example: an application of Armstrong relations, J. Comput. Syst. Scien. 33 (1986) 125-141.

[18] Mannila H., Raiha K. J., Practical algorithms for finding prime attributes and testing normal forms, Processings of the eighth ACM SIGACT-SIGART Syposium on Principles of Database Systems Philadelphia, PA (ACM, New York, 1989), p. 128-133.

[19] Osborn S. L., Testing for existence of a covering Boyce-Codd normal form, Infor. Proc. Lett. 8 (1) (1979) 11-14.

[20] Thi V.D., Investigation on Combinatorial Characterzation Related to Functional Dependency in the Relational Datamodel (in Hungarian), MTA-SZTAKI Tanulmanyok, 191 (1986) 1-157, Ph.S. Dissertation.

[21] Thi V.D., Minimal keys and antikeys, Acta Cybernica Hungary 7 (4) (1986) 361-371.

[22] Thi V.D., On antikeys in the relational datamodel (in Hungarian), Alkalmazott Matematikai Lapok 12 (1986) 111-124.

[23] Tsou D. M., Fischer P.C., Decomposition of a relation scheme into Boyce-Codd normal form, SIGACT NEWS 14 (1982) 23-29.

Vu Duc Thi - Institute of Information Technology.

Received December 14, 1998

Luong Cao Son - Informatic Centre of Office of Government. 